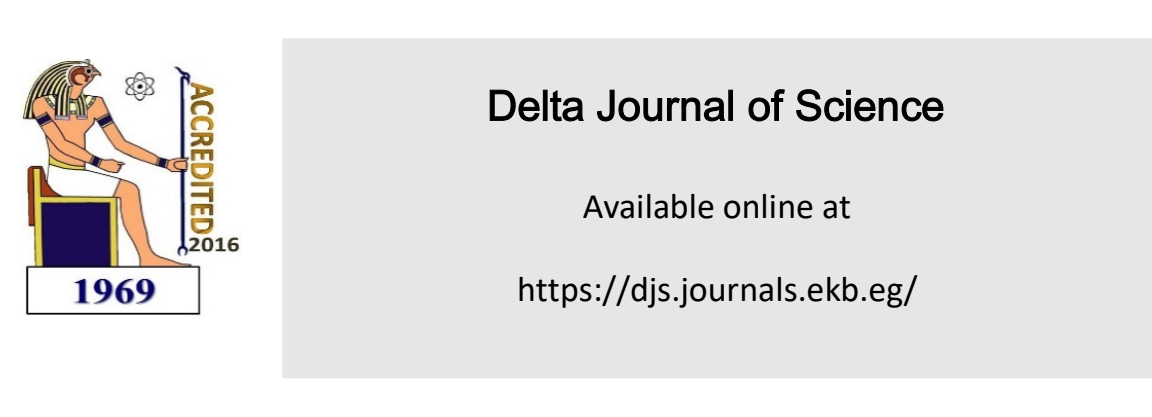

Research Article

MATHEMATICS

\title{
Higher estimates for the solution of a heavy rigid body moving under the action of a Newtonian force field
}

\author{
T. S. Amer ${ }^{1}$ and I. M. Abady ${ }^{2}$
}

${ }^{1}$ Mathematics Department, Faculty of Science, Tanta University, Tanta 31527, Egypt.

${ }^{1}$ Email:tarek.amer@science.tanta.edu.eg, $\underline{\text { tarekamer30@hotmail.com }}$

${ }^{2}$ El-Wastania, Kafr El-Dawar, Behira 22635, Egypt

2Email: abady47@yahoo.com

\begin{abstract}
In this paper, we introduce a new solution of the Euler's dynamic equations for the rotational motion of a rigid body about a fixed point under the action of a Newtonian force field. The components of the angular velocity vector for this solution are differing from the most famous cases. We assumed that the center of mass of the rigid body coincides with the fixed point and a restriction on an initial condition is applied. The obtained solution is represented graphically using most recent computer codes to describe the motion at any time and is considered as a modification of Euler's case.
\end{abstract}

Key words: Euler's equations, Rigid body motion, Newtonian field

\section{Introduction:}

The rotational motion of a rigid body about a fixed point in a Newtonian force field is one of the important problems in theoretical classical mechanics. This problem attracted the interest of many researchers during the last five decades e. g. [1-7]. The great importance of this research subject is due to the wide range of its applications in mechanics. To solve these problems we need to deal with intricate techniques because they are governed by a system contains six non-linear differential equations besides with three first integrals [8]. The exact solutions of such systems require an additional fourth algebraic first integral. Many researches realized such integral for famous special cases, which have some restrictions on the body center of mass location and on the torques acting on the body [9].
The perturbed rotational motion of a heavy solid close to regular precession with constant restoring moment was treated in [2] and [3]. The authors assumed some initial conditions to achieve the analytical solutions of the equations of motion using averaging method [10] up to the first and second approximations. The rotatory motion of a symmetric gyrostat about a fixed point when one component of the gyrostatic torque is applied and in the presence of some torques was considered in [4] and generalized in [5]. The motion of an electromagnetic gyroscope is investigated in [6] when a Newtonian field, perturbed moments and restoring ones are applied. The averaging technique [10] is used to obtain the first order approximate analytical solutions. The graphical representations of these solutions are presented to describe the motion at any instant. The

Higher estimates for the solution of a heavy rigid body movingunder the action of a Newtonian force field 


\section{T. S. Amer ${ }^{1}$ and I. M. Abady ${ }^{2}$}

rotational motion of that body under the action of a Newtonian force field with the application of the third component of a gyrostatic moment is investigated in [7]. The approximate periodic solutions of the governing equations are obtained using the small parameter method of Poinacré [11]. This method and its modifications [12-13] are used in [14] to construct the periodic solutions of limiting case for the motion of a rigid body about a fixed point in a Newtonian force field.

The rotational motion of a heavy solid about a fixed point in the presence of a gyrostatic moment vector is presented in [15]. The authors supposed that the body has rapidly spinning about the major or the minor principal axis of the ellipsoid of inertia. Krylov-Bogoliubov-Mitropolski technique [10] is modified and used to achieve the periodic solutions of the equations of motion.

The perturbed self-excited rigid body problem with a fixed point is investigated in [16]. The averaging theory [17] is used to study the periodic orbits up to first order. In [18], the authors presented the possibility of constructing exact analytic solutions concerning the dynamic Euler equations of motion.

The spinning motion of the hovering magnetic top and its dynamic stability were analyzed in [19] and [20]. The numerical integration of a heavy magnetic top is investigated in [21].

Existence of periodic motions of a rigid body was investigated in [22]. The small parameter method was used to obtain the periodic solutions of the equations of motion. The center of mass of the body is slightly shifted from a dynamically symmetric axis. The generalization of this problem was treated in [23] when the body rotates under the action of a Newtonian field and in the presence of one component of the gyrostatic moment vector. A new exact solution of the equations of motion of a rigid body is investigated in [24] when the body moves under the action of a uniform force field. The author assumed that the center of mass of the body is located at meridional plane and the principal torques of inertia satisfied a simple algebraic condition.

In this work, we extend the previous studies when the rigid body moves under the action of a Newtonian force field arising from an attracting center located on the downward fixed axis. We assume that the center of mass of the body coincides with the fixed point (origin). The achieved solution is obtained after taking account some algebraic assumptions concerning on the moments of inertia. This solution is represented graphically, in the rest of this paper, to show the behavior of the body motion under the action of Newtonian force field. From this point of view, the current study may be regarded as a modification of Euler's case for the motion of a rigid body.

\section{Equations of motion}

Consider the motion of a heavy rigid body that rotates about a fixed point $O$, in the body, under the influence of a Newtonian force field arising from an attracting center $O_{1}$ being located on a downward fixed axis passing through the fixed point $O$. Let $O X Y Z$ be a fixed coordinate system and another moving one $O x y z$ which is fixed in the body and whose axes are directed along the principal axes of inertia of the body with origin $O$. The equations of motion are given below [14]

$$
\begin{aligned}
& A \dot{p}+(C-B) q r=M g\left(\gamma_{2} z_{0}-\gamma_{3} y_{0}\right)+N(C-B) \gamma_{2} \gamma_{3}, \\
& B \dot{q}+(A-C) r p=M g\left(\gamma_{3} x_{0}-\gamma_{1} z_{0}\right)+N(A-C) \gamma_{3} \gamma_{1}, \\
& C \dot{r}+(B-A) p q=M g\left(\gamma_{1} y_{0}-\gamma_{2} x_{0}\right)+N(B-A) \gamma_{1} \gamma_{2},
\end{aligned}
$$

with

$\dot{\gamma}_{1}=r \gamma_{2}-q \gamma_{3}, \quad \dot{\gamma}_{2}=p \gamma_{3}-r \gamma_{1}$,

$\dot{\gamma}_{3}=q \gamma_{1}-p \gamma_{2}$,

where $A, B$ and $C$ are the principal moments of inertia of the body; $p, q$ and $r$ are the projections of the angular velocity $\underline{V}$ of the body on the principal axes of inertia; $\underline{\gamma}=\left(\gamma_{1}, \gamma_{2}, \gamma_{3}\right)$ is the unit vector in the direction of the $Z$-axis; $M$ is the mass of the body; $g$ is the gravitational acceleration; $x_{0}, y_{0}$ and $z_{0}$ are the coordinates of the center of mass in the moving coordinate system Oxyz. The overdot here refers to differentiation with respect to the time $t$ and $N=\left(3 \lambda / R^{3}\right)$ where $R$ is the distance from the fixed point $O$ to the attracting center $O_{1}$ and $\lambda$ is the coefficient of such center.

Equations (1) and (2) admit the following three first integrals

$$
\begin{aligned}
& \gamma_{1}^{2}+\gamma_{2}^{2}+\gamma_{3}^{2}=1, \\
& A p \gamma_{1}+B q \gamma_{2}+C r \gamma_{3}=C_{0}, \\
& \left(A p^{2}+B q^{2}+C r^{2}\right)+2 M g\left(x_{0} \gamma_{1}+y_{0} \gamma_{2}\right. \\
& \left.+z_{0} \gamma_{3}\right)+N\left(A \gamma_{1}^{2}+B \gamma_{2}^{2}++C \gamma_{3}^{2}\right)=2 C_{1},
\end{aligned}
$$

where $C_{0}$ and $C_{1}$ are constants.

\section{Euler's case}




\section{T. S. Amer ${ }^{1}$ and I. M. Abady ${ }^{2}$}

As in Euler's case, we obtain the following first fourth integral according to the presence of Newtonian field

$$
\begin{aligned}
& A^{2} p^{2}+B^{2} q^{2}+C^{2} r^{2}-N\left(B C \gamma_{1}^{2}\right. \\
& \left.+C A \gamma_{2}^{2}+A B \gamma_{3}^{2}\right)=C_{0}^{2} .
\end{aligned}
$$

Making use of the first two integrals in (3) and the fourth integral (4), one obtains

$$
\gamma_{1}=\frac{A p}{C_{0}}, \quad \gamma_{2}=\frac{B q}{C_{0}}, \quad \gamma_{3}=\frac{C r}{C_{0}} \text {. }
$$

Substituting from systems (1) and (2) into the third equation of system (3), one gets

$$
\begin{aligned}
& C_{2}\left[\left(N^{*} A^{2}+1\right) A p^{2}+\left(N^{*} B^{2}+1\right) B q^{2}+\left(N^{*} C^{2}+1\right) C r^{2}\right] \\
& +x_{0} A p+y_{0} B q+z_{0} C r=C_{3},
\end{aligned}
$$

where

$$
C_{2}=\frac{C_{0}}{2 M g}, \quad C_{3}=\frac{C_{0} C_{1}}{M g}, \quad N^{*}=\frac{N}{C_{o}^{2}} .
$$

Equation (6) represents a linear combination of the first integrals (3), the fourth integral (4) and (5). So, we seek for a solution that satisfies the previous equation (6).

\section{The modified solution}

For our scope, let us consider the following choice together with the assumptions of Euler's case

$$
A>B>C \text {. }
$$

This choice allows us to rewrite equation (6) in the form

$$
p^{2}=\frac{2 C_{1}-N_{2}^{*} B q^{2}-N_{3}^{*} C r^{2}}{N_{1}^{*} A},
$$

where

$N_{1}^{*}=N^{*} A^{2}+1, \quad N_{2}^{*}=N^{*} B^{2}+1, \quad N_{3}^{*}=N^{*} C^{2}+1$.

Substituting from (7) into (4), we can obtain directly $q^{2}$ in the form

$q^{2}=C_{4}-C_{5} r^{2}$.

Here,

$$
\begin{aligned}
& C_{4}=\frac{\left[C_{0}^{2}-\left(2 C_{1} A / N_{1}^{*}\right)+\left(2 C_{1} N^{*} A B C / N_{1}^{*}\right)\right]}{\left[1-N^{*} A C-\left(N_{2}^{*} A / N_{1}^{*} B\right)+\left(N^{*} N_{2}^{*} A C / N_{1}^{*}\right)\right] B^{2}}, \\
& C_{5}=\frac{\left[1-N^{*} A B-\left(N_{3}^{*} A / N_{1}^{*} C\right)+\left(N^{*} N_{3}^{*} A B / N_{1}^{*}\right)\right] C^{2}}{\left[1-N^{*} A C-\left(N_{2}^{*} A / N_{1}^{*} B\right)+\left(N^{*} N_{2}^{*} A C / N_{1}^{*}\right)\right] B^{2}} .
\end{aligned}
$$

The substitution from (8) into (7) gives

$$
\begin{aligned}
& p^{2}=C_{6}+C_{7} r^{2} ; \\
& C_{6}=\left(2 C_{1}-N_{2}^{*} B C_{4}\right) / N_{1}^{*} A, \\
& C_{7}=\left(N_{2}^{*} B C_{5}-N_{3}^{*} C\right) / N_{1}^{*} A .
\end{aligned}
$$

Substituting from equalities (8) and (9) into the third one of the system of equations (1), we get

$$
\int \frac{d r}{[(A-B) / C] \sqrt{\left(C_{6}+C_{7}\right)\left(C_{4}-C_{5}\right)} r^{2}}=\int d t .
$$

Under the present circumstances, the solution of the previous integration can be obtained easily as

$$
r=\left\{k-[(A-B) / C] \sqrt{\left(C_{6}+C_{7}\right)\left(C_{4}-C_{5}\right)} t\right\}^{-1} ; k=\text { const }
$$

An inspection of equations (8), (9) and (10), broadly speaking, provides the solution of the problem when the rigid body rotates under the action of a Newtonian force field. This elucidates that, we can separately determine the components of the angular velocity vector $p, q$ and $r$ as functions of time $t$ from these equations. Consequently, we can obtain directly the scalar value of the angular velocity vector in the form

$$
V=|\underline{V}|=\left|\sqrt{\left(C_{4}+C_{6}\right)+\left[\frac{1+C_{7}-C_{5}}{\left(k-C_{8} t\right)^{2}}\right]}\right|,
$$

where

$$
C_{8}=[(A-B) / C] \sqrt{\left(C_{6}+C_{7}\right)\left(C_{4}-C_{5}\right)} .
$$

\section{Discussion of results}

In this section, our aim is to provide some numerical results using the computer programs. The following data are used to determine the motion in the considered problem

$$
\begin{aligned}
& A=7 \mathrm{~kg} \cdot \mathrm{m}^{2}, B=6 \mathrm{~kg} \cdot \mathrm{m}^{2}, C=4 \mathrm{~kg} \cdot \mathrm{m}^{2}, M=100 \mathrm{~kg}, \\
& g=9.8 \mathrm{~m} / \mathrm{s}^{2}, N=\left(200400,500 \mathrm{~kg} \cdot \mathrm{m} / \mathrm{s}^{2}\right.
\end{aligned}
$$

Figures (1-4) show the variation of the angular velocity $V$ versus time $t$ in 2-D plane when $N=200 \mathrm{~kg} . \mathrm{m} / \mathrm{s}^{2}$ and $N=400 \mathrm{~kg} \cdot \mathrm{m} / \mathrm{s}^{2}$. It is to be noted that, the value of the angular velocity of the body monotonically increases with the increase in time (see figures 1, 3) till it has attained its maximum value whenever $t=k / C_{8}$, i.e. when the dominator of the second bracket in equation (11) vanishes, at different values of Newtonian force field. The domain of equation (11) is $\mathfrak{R}^{+} \cup\{0\}-\left\{k / C_{8}\right\}$ and its range is $\mathfrak{R}^{+} \cup\{0\}$, 


\section{T. S. Amer ${ }^{1}$ and I. M. Abady ${ }^{2}$}

where $\mathfrak{R}^{+}$is the positive real numbers, $\left(C_{4}+C_{6}\right)>0$ and $\left(1+C_{7}-C_{5}\right)>0$.

Above the value $t=k / C_{8}$, the numerical computations show that the angular velocity gradually decreases as the time goes on, in a similar manner to its increase, (see figures 2, 4). Further, we observe that the growth in the value of Newtonian force field leads to increase in $t$ and $V$ as well.

To make the results more favor, we proceed to illustrate the numerical results in 3-D space. Figures (5-7) and (8-10) represent the behavior of the angular velocity $V$ and time $t$ via $\xi=(V-t)$ when the Newtonian force field equals to 200 and 400, respectively. It should be noticed that figures $(5,8)$, $(6,9)$ and $(7,10)$ describe the behavior of the body below, near and above the maximum value of time $t$, respectively. The spatial figures for most values of Newtonian force field are presented; see figures (1116).

It is clear from all previous figures that, the Newtonian force field has acquired a significant influence on the behavior of our model. Such results may be utilized in many industrial applications in various fields; like satellite, spacecraft and manipulators.
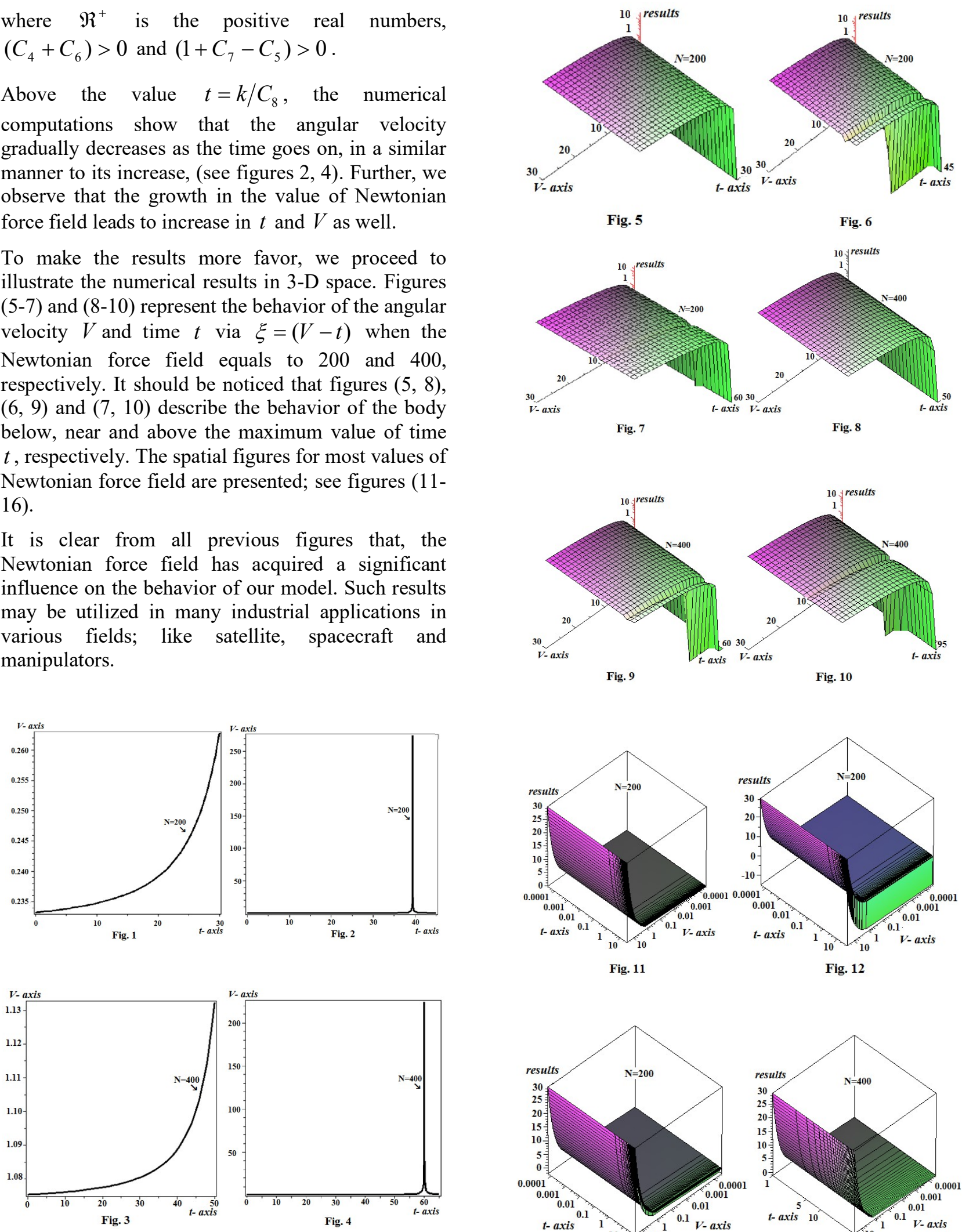

Fig. 9

Fig. 10

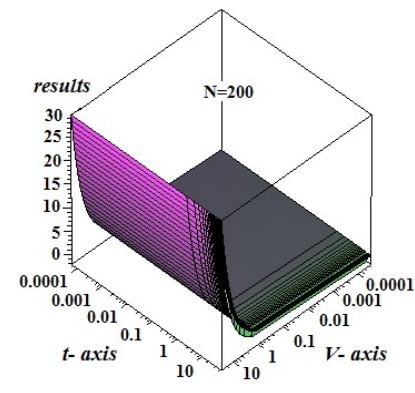

Fig. 13

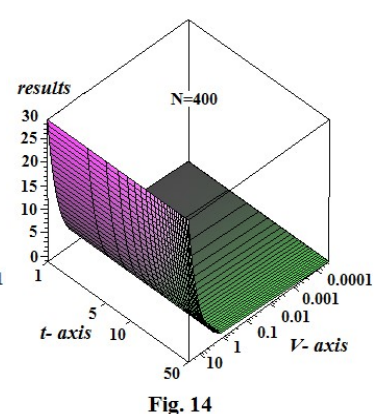

Fig. 14 
T. S. Amer ${ }^{1}$ and I. M. Abady ${ }^{2}$
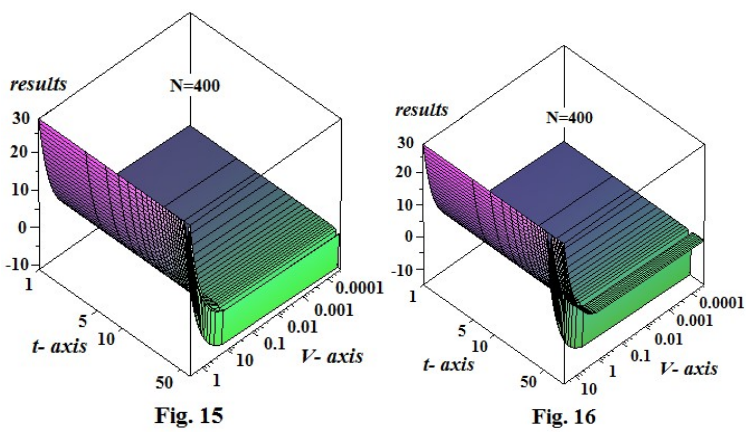

\section{Conclusion}

In this work, we have developed a modified solution, represented by (8)-(10), for the Euler's dynamic equations (1) with the aid of Poinsot's equations (2), when the rigid body rotates under the action of a Newtonian force field. The obtained angular velocity components are different from Lagrange's case, Kovaleveskaya's case, Euler's case (when the body rotates without any applied torques) or from any special case. A restriction on the choosing of initial conditions of $p(0), q(0), r(0), \gamma_{1}(0), \gamma_{2}(0)$ or $\gamma_{3}(0)$ according to the meaning of $C_{0}^{2}$ is considered. The obtained solution is considered as a modification for both Euler's case and Ershkov [24] work when the Newtonian filed has no effect, i.e. vanishes. The graphical representations of the obtained angular velocity solution are presented through different figures. The numerical results have shown that the Newtonian force field value has an important effect on the rigid body motion. However, the analytical results of the rotational motion of a rigid body about a fixed point can be exploited in industrial applications, such as satellites, autopilots and aircrafts.

\section{References}

[1] Leimanis, E., The general problem of the motion of coupled rigid bodies about a fixed point, Springer Verlag, Berlin, 1965.

[2] Leshchenko, D. D., and Sallam, S. N., Perturbed rotational motions of a rigid body similar to regular precession, J. Appl. Math. Mech. 54, 2, 183-190, 1990.

[3] Leshchenko, D. D., On the evolution of rigid body rotations, Int. Appl. Mech 35, 1, 93-99, 1999.

[4] Ismail, A. I., Amer, T. S., and Shaker, M. O., Perturbed motions of a rotating symmetric gyrostat, Engng. Trans. 46, 3-4, 271-289, 1998.

[5] Amer, T. S., New treatment of the perturbed motions of a rotating symmetric gyrostat about a fixed point, Thai J Math., 2009.
[6] Ismail, A. I., Amer, T. S., El Banna, S. A., and El-Ameen M. A., Electromagnetic gyroscopic motion, J. Appl. Math., 1-14, 2012.

[7] Ismail, A. I., and Amer, T. S., The fast spinning motion of a rigid body in the presence of a gyrostatic momentum $\ell_{3}$, Acta Mech. 154, 31-46, 2002.

[8] Borisov, A. V., and Mamaev, I. S., Rigid body dynamics, Izhevsk: RCD, 2001.

[9] Romano, M., Exact analytic solution for the rotation of a rigid body having spherical ellipsoid of inertia and subjected to a constant torque, Celest. Mech. Dyn. Astr. 100,181-189, 2008.

[10] Bogoliubov, N. N., and Mitropolski, Yu. A., Asymptotic methods in the theory of non-linear oscillations, Gordon and Breach, New York, 1961.

[11] Nayfeh, A. H., Perturbations methods, WILEYVCH Verlag GmbH \& Co. KGaA, Weinheim, 2004.

[12] Proskuriakov, A. P., Periodic oscillations of quasilinear autonomous systems with two degrees of freedom, J. Appl. Math. Mech. 24, 6, 1098-1104, 1960.

[13] Proskuriakov, A. P., On the construction of periodic solutions of quasi-linear autonomous systems with several degrees of freedom, J. Appl. Math. Mech. 26, 2, 519-528, 1962.

[14] El-Barki, F. A., and Ismail, A. I., Limiting case for the motion of a rigid body about a fixed point in the Newtonian force field, Z. Angew. Math. Mech. 75, 11, 821-829, 1995.

[15] Amer, T., Ismail, A., and Amer, W., Application of the Krylov-Bogoliubov-Mitropolski technique for a rotating heavy solid under the Influence of a gyrostatic moment, J. Aerospace Eng. 25, 3, 421-430, 2012.

[16] Ferrándiz, J. M., Guirao, J. L. G., and Vera, J. A., A note on the periodic orbits of a self excited rigid body, Mech. Res. Commun. 56, 50-52, 2014.

[17] Llibre, J., Averaging theory and limit cycles for quadratic systems, Radovi Matematicki 11, 215-228, 2002.

[18] Panayotounakos, D. E., Rizou, I., and Theotokoglou, E., A new mathematical construction of the general nonlinear ODEs of motion in rigid body dynamics (Euler's equations), Appl. Math. Comput. 217, 8534-8542, 2011.

[19] Flanders, P., Gov S., Shtrikman S., and Thomas $\mathrm{H}$., On the spinning motion of the hovering magnetic top, Phys. D 126, 225-235, 1999. 


\section{T. S. Amer ${ }^{1}$ and I. M. Abady ${ }^{2}$}

[20] Gov, S., Shtrikman, S., and Thomas, H., On the dynamical stability of the hovering magnetic top, Phys. D 126, 214-224, 1999.

[21] Miguel, A. S., Numerical integration for the dynamics of the heavy magnetic top, Phys. Lett. A 335, 235-244, 2005.

[22] Elfimov, V. S., Existence of periodic solutions of equations of motion of a solid body similar to the Lagrange gyroscope, J. Appl. Math. Mech. 42, 2, 251-258, 1978.

[23] Ismail, A. I., Sperling, L. and Amer, T. S., On the existence of periodic solutions of a gyrostat similar to Lagrange's gyroscope, Technische Mechanik 20, 4, 295-304, 2000.
[24] Ershkov, S. V., New exact solution of Euler's equations (rigid body dynamics) in the case of rotation over the fixed point, Arch. Appl. Mech. 84, 385-389, 2014.

\section{تقديرات أعلى لحل جسم متماسك ثقيل يتحرك تحت تأثير مجال نيوتوني}

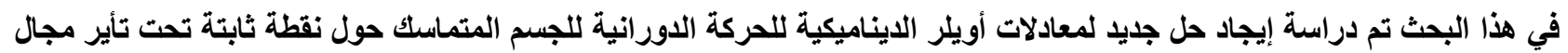

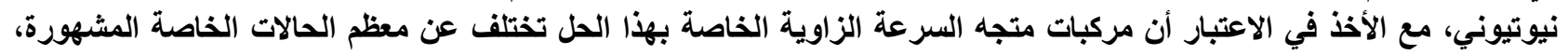

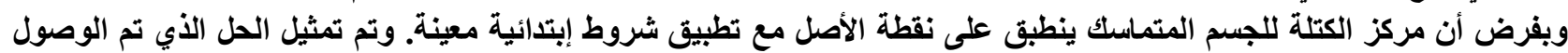
إليه هندياً باستخدام برامج حديثة وذلك لوصف الحتكة الحكة عند أية لحظة زمنية، ويعتبر هذا الحل تعديلاً لحالة أويلر. 